\title{
Improved Semantic Representation for Domain-Specific Entities
}

\author{
Mohammad Taher Pilehvar and Nigel Collier \\ Language Technology Lab \\ Department of Theoretical and Applied Linguistics \\ University of Cambridge \\ Cambridge, UK \\ $\{$ mp 792, nhc30\}@cam.ac.uk
}

\begin{abstract}
Most existing corpus-based approaches to semantic representation suffer from inaccurate modeling of domain-specific lexical items which either have low frequencies or are non-existent in open-domain corpora. We put forward a technique that improves word embeddings in specific domains by first transforming a given lexical item to a sorted list of representative words and then modeling the item by combining the embeddings of these words. Our experiments show that the proposed technique can significantly improve some of the recent word embedding techniques while modeling a set of lexical items in the biomedical domain, i.e., phenotypes.
\end{abstract}

\section{Introduction}

Semantic representation is one of the oldest, yet most active, research areas in Natural Language Processing (NLP) owing to the central role it plays in many applications (Pilehvar and Navigli, 2015). The field has experienced a resurgence of interest in recent years with the introduction of lowdimensional continuous space models that leverage neural networks for learning semantic representations. Word2vec (Mikolov et al., 2013) is a good example which despite its recent invention has found its way prominently into literature, mainly thanks to its ability to be quickly and effectively trained on large amounts of text.

However, since most of these corpus-based techniques base their representation only on the co-occurrence statistics derived from text corpora, they fall short of effectively modeling lexical items for which not many statistical clues can be obtained from the underlying corpus. Several attempts have been made to improve word embed- dings with the help of knowledge derived from other resources (Yu and Dredze, 2014; Bian et al., 2014; Faruqui et al., 2015) or by including arbitrary contexts in the training process (Levy and Goldberg, 2014). However, most of these techniques still suffer from another deficiency of word embeddings that they inherit from their countbased ancestors: they conflate the different meanings of a word into a single vector representation. Attempts have been made to tackle the meaning conflation issue of word-level representations. A series of approaches cluster the context of a word prior to representation (Reisinger and Mooney, 2010; Huang et al., 2012; Neelakantan et al., 2014) whereas others exploit lexical knowledge bases for sense-specific information (Rothe and Schütze, 2015; Chen et al., 2014; Iacobacci et al., 2015; Camacho-Collados et al., 2015).

We propose a model that addresses both these issues through a mapping of a lexical item to a sorted list of representative words that brings about two advantages. Firstly, it pinpoints with an inherent disambiguation the meaning of the given lexical item at a deeper semantic level. Secondly, by casting the representation of the item as that of a set of potentially more frequent words, our approach can provide a more reliable representation of domain-specific items based on significantly more statistical knowledge. Our experiments show that the proposed model can provide a considerable improvement over some of the stateof-the-art word embedding approaches in a semantic similarity-based task.

Data. The final goal of this paper is to improve the semantic representation of domain-specific terms and phrases which usually have low frequencies (or are non-existent) in open-domain corpora and hence have a lower chance of being effectively modeled by existing word representation 
techniques. Therefore, for our experiments we retrieved terms and phrases from a domain-specific ontology in the biomedical domain. Specifically, as our dataset in the experiments we opted for Human Phenotype Ontology (Sebastian Khler, 2014, HPO) which is a standardized vocabulary of phenotypic abnormalities encountered in human disease. Semantic modeling of phenotypes has several applications in the biomedical domain such as profiling heritable diseases or understanding the genetic origins of diseases (Collier et al., 2013).

\section{Improved Semantic Representation}

In this section we explain how our technique builds on top of pre-trained word embeddings to provide a more accurate semantic representation.

\subsection{Disambiguation}

As mentioned in the Introduction, one of the drawbacks of word-level representations is that they conflate different meanings of a word into a single vector. Our technique constructs a more accurate semantic representation of a lexical item by constraining its semantics through a set of relevant words. Interestingly, we achieve this on the basis of the same set of word-level representations. To this end, we first disambiguate the content word(s) in a given lexical item. In our experiments, we used Babelfy (Moro et al., 2014) which is a state-of-the-art WSD system based on the BabelNet sense inventory. BabelNet is a merger of Wikipedia and WordNet, among other resources (Navigli and Ponzetto, 2012). Let $t=$ flexion contracture of digit be the phrase we are interested in modeling. The disambiguation phase transforms the phrase to three BabelNet concepts corresponding to the intended meanings of the content words $\{$ flexion, contracture, and digit $\}$. Disambiguating with respect to BabelNet provides us with an additional benefit: it links a content word to the corresponding Wikipedia page of its intended meaning, giving us the chance to draw additional context for improving its representation.

\subsection{Representative list}

Let the set of disambiguated concepts for a lexical item $t$ be $\mathcal{C}_{t}$. We further enrich this set by adding all the BabelNet concepts that have a semantic link (in the semantic network of BabelNet) to any of the concepts in $\mathcal{C}_{t}$. Let the enriched set of concepts be $\mathcal{C}_{t}^{*}$. Our goal here is to map
$\mathcal{C}_{t}^{*}$ to a set of most relevant words that can represent its semantics. We achieve this by exploiting the fact that these concepts are linked to relevant Wikipedia articles. Let $D_{t}$ be the set of Wikipedia articles retrieved for $t$ (i.e., the set of articles that are associated with the concepts in $\mathcal{C}_{t}^{*}$ ). We analyze the textual content of these articles by leveraging the method proposed by Camacho-Collados et al. (2015) and retrieve a sorted list of salient words. Specifically, we use lexical specificity and contrast word frequency statistics between $D_{t}$ and all articles in Wikipedia. Lexical specificity (Lafon, 1980) is a statistical measure based on the hypergeometric distribution which can be used to compute the semantic importance of an arbitrary vocabulary word $w$ for $D_{t}$ as:

$$
\operatorname{Spec}(H ; h ; G ; g)=-\log _{10} P(X \geq g)
$$

where $H$ and $h$ are the respective aggregate frequencies of all words in all Wikipedia articles and $D_{t}$, and $G$ and $g$ are the respective frequencies of $w$ in all Wikipedia articles and $D_{t}$. For a given lexical item $t$, we construct the set of semantically representative words $\mathcal{R}_{t}$ by keeping the words that are relevant to $D_{t}$ with a minimum confidence of $99 \%$ according to the hypergeometric distribution, i.e., $P(X \geq 0.01)$.

For our example phenotype flexion contracture of digit, the representative list $\mathcal{R}_{t}$ comprises of around 1300 weighted words, with the top ones being muscle, finger, spasticity, toe, hand, patient, and spastic. Please note that our technique mapped an ambiguous term digit to a set of more semantically constrained keywords such as finger, toe, and hand. This enables us to construct a sense-specific representation of the word by leveraging word-level representations.

\subsection{Vector construction}

So far, we mapped a given lexical item $t$ to a set of relevant concepts $\mathcal{C}_{t}^{*}$ and obtained for this set the sorted list $\mathcal{R}_{t}=\left\{r_{1}, \ldots, r_{m}\right\}$ of the most semantically representative words. The final step is to construct a vector representation $V_{t}$ for $t$. We do this by combining the vectors for the words in $\mathcal{R}_{t}$. Let $\mathcal{V}(x)$ be the vector representation given by a model such as Word2vec for the word $x$. We compute the weight for the $i^{t h}$ dimension of the vector $V_{t}$, i.e., $v_{i}$, as:

$$
v_{i}=\sum_{j=1}^{m} e^{-\lambda j} \mathcal{V}\left(r_{j}\right)_{i} \quad i=1, \ldots, n
$$




\begin{tabular}{|c|c|c|c|c|c|}
\hline sim. & Flexion contracture of digit & sim. & Bipolar affective disorder & sim. & Chaotic rapid conjugate ocular movements \\
\hline 0.94 & Flexion contracture of finger & 0.80 & Personality disorder & 0.85 & Abnormal conjugate eye movement \\
\hline 0.92 & Flexion contracture of thumb & 0.85 & Schizophrenia & 0.80 & Jerky ocular pursuit movements \\
\hline
\end{tabular}

sim. Hydranencephaly (A defect of development of the brain characterized by replacement of greater portions of the cerebral hemispheres [...].)

0.81 Porencephaly (A disorder of the brain in which a cyst or cavity filled with cerebrospinal fluid develops in the cerebral hemisphere.)

0.79 Dandy-walker malformation (A congenital brain malformation typically characterized by incomplete formation of the cerebellar vermis, dilation of [...].)

0.77 Ventriculomegaly (An increase in size of the ventricular system of the brain.)

Table 1: The most similar phenotypes (among 11,591) to four phenotypes in the HPO database together with their similarity scores. We also show the definitions for more technical terms in parentheses.

where $\mathcal{V}\left(r_{j}\right)_{i}$ is the weight of the $i^{\text {th }}$ dimension of the base vector for the $j^{\text {th }}$ word in $\mathcal{R}_{t}$ and $e^{-\lambda j}$ is a decay function (with the decay constant $\lambda$ ) that gives more importance to the higher ranking terms in $\mathcal{R}_{t}$. In our experiments, we did not perform a tuning on the value of $\lambda$ which was set to $\frac{1}{5}$. Please note that the dimensionality of $V_{t}$ is identical to that of the base word representations, i.e., $n$. Table 1 shows the top-3 most similar phenotypes for four phenotypes in the HPO ontology when Word2vec was used as the base representation.

\section{Experiments}

We evaluate our model in the semantic representation of phenotypes in the HPO ontology.

\subsection{Dataset}

As of February 2016, the HPO ontology comprises of 11,591 phenotypic abnormalities. Each of these concepts is provided with a title (with an average length of four words) and about $35 \%$ of all these concepts are associated with synonymous titles (by average, each of these concepts has 1.94 synonyms). For example, Keratoconjunctivitis sicca is a phenotype for which three synonymous titles are provided by the ontology: Dry eye syndrome, Keratitis sicca, and Xerophthalmia.

\subsection{Tasks}

Based on the ontological structure of HPO, we propose two tasks in the framework of semantic similarity measurement.

Synonym identification. Let $\mathcal{P}$ be the set of all phenotypes in the HPO ontology. Let $\mathcal{P}^{*}=$ $\left\{p_{1}, \ldots, p_{k}\right\}(\subset \mathcal{P})$ be the subset of $k$ phenotypes for which at least one synonymous phenotype is provided in HPO and $\mathcal{S}_{p_{i}}=\left\{s_{p_{i}}^{1}, \ldots, s_{p_{i}}^{l}\right\}$ be the set of $l$ synonymous phenotypes for phenotype $p_{i}$. Given a $s_{p_{i}}$, the task here is simply to identify the corresponding phenotype (i.e., $p_{i}$ ). In other words, the system has to identify the set of synonymous phenotypes to a given phenotype. Specifically, we compare the representation of $s_{p_{i}}$ with those of all the phenotypes in $\mathcal{P}$, obtaining a sorted list of most similar phenotypes. Ideally, the concept containing the synonymous title should appear at the top of this list. The higher the rank of $p_{i}$ for a given $s_{p_{i}}$, the better has the system captured the semantics of the phenotypes. For this task we have 7193 synonymous titles $\left(\sum_{i=1}^{k}\left|\mathcal{S}_{p_{i}}\right|\right)$ that are to be matched with their corresponding phenotypes (among a total of 11,591 phenotypes).

Hypernym identification. Similarly to the previous experiment, a system's task here is to identify the hypernym of a given phenotype. The aim of this experiment is to have a broader evaluation that can also cover all those concepts that do not provide synonymous titles (the dataset comprises of 11,590 phenotypes that have a hypernym).

\subsection{Baselines}

As baseline, we benchmark our improved representations against Word2vec. We use the 300dimensional vectors trained on the Google News corpus (about 100B tokens). We also report results for the Word2vec vectors when retrofitted using the approach of Faruqui et al. (2015) to the Paraphrase Database (Ganitkevitch et al., 2013, PPDB) and SNOMED-CT ${ }^{1}$. The latter is a comprehensive clinical terminology from which we extracted $108 \mathrm{~K}$ synonymous sets, each comprising an average of 2.7 synonyms. We also compare our representations against the 300-dimensional GloVe vectors (Pennington et al., 2014) trained on the Wikipedia 2014 + Gigaword 5 corpus (6B tokens).

We were also interested in verifying how Word2vec and GloVe would perform if trained on

\footnotetext{
${ }^{1}$ https: / / www.nlm.nih.gov/snomed/
} 


\begin{tabular}{|c|c|c|c|c|}
\hline System & Description & Mean rank & Median rank & First match \\
\hline Word2vec & \multirow{2}{*}{ Trained on open-domain data (Google News) } & 1343.6 & 11 & $22 \%$ \\
\hline Word2vec (2nd order) & & 664.1 & 6 & $28 \%$ \\
\hline Word2vec & \multirow{2}{*}{ Trained on in-domain data (PubMed) } & 224.1 & 4 & $32 \%$ \\
\hline Word2vec (2nd order) & & 198.2 & 3 & $36 \%$ \\
\hline GloVe & \multirow[b]{2}{*}{ Trained on open-domain data (Wikipedia + Gigaword) } & 1326.4 & 9 & $24 \%$ \\
\hline GloVe (2nd order) & & 673.5 & 6 & $28 \%$ \\
\hline GloVe & \multirow{2}{*}{ Trained on in-domain data (PubMed) } & 701.4 & 4 & $34 \%$ \\
\hline GloVe (2nd order) & & 493.5 & 3 & $36 \%$ \\
\hline Word2vec & Trained on Google News, retrofitted to PPDB & 1357.4 & 8 & $26 \%$ \\
\hline Word2vec & Trained on Google News, retrofitted to SNOMED-CT & 1346.2 & 9 & $25 \%$ \\
\hline Random baseline & Random selection of the synonymous phenotype & 5473.0 & 5473.0 & $0 \%$ \\
\hline
\end{tabular}

Table 2: Evaluation results for the synonym identification task. We report mean and median rank (lower better) and the percentage of phenotypes for which the rank was equal to one (first match; higher better).

an in-domain corpus. Thankfully, the biomedical domain is a rich domain for which large amounts of textual data are available. We retrieved a corpus of $4 \mathrm{~B}$ tokens from article abstracts indexed in PubMed $^{2}$. We then trained Word2vec and GloVe with window size of 5 words and the same dimensionality as the open-domain vectors (i.e., 300). For Word2vec we opted for the skip-gram model.

\subsection{Results and discussion}

Table 2 shows the evaluation results. We report mean and median rank of the target phenotype in the sorted list of most semantically similar phenotypes as well as the percentage of target phenotypes for which this rank was equal to one, i.e., the synonymous title was computed as the most similar item (first match in the table). As a reference, we also report the performance of a baseline which randomly picks the target phenotype.

We can see that a considerable performance improvement was gained when our technique was used for improving Word2vec and GloVe representations trained on open-domain corpora. Interestingly, even when the vectors were trained on an in-domain corpus (PubMed) that covers a large portion of the phenotypes with high frequencies, our model was still able to provide statistically significant improvements according to mean rank over the vanilla Word2vec and GloVe. ${ }^{3}$ The retrofitting of the vanilla vectors improved median rank and first match irrespective of the resource but did not match the performance of our model.

The substantial improvement of our approach in the open-domain setting should be attributed to

\footnotetext{
${ }^{2}$ http://www.ncbi.nlm.nih.gov/pubmed/

${ }^{3}$ According to $t$-test with $95 \%$ confidence interval.
}

its mapping of domain-specific phenotypes with lower frequencies to a set of more frequent representative terms. In fact, only around $60 \%$ of the unique tokens of the phenotypes in the HPO ontology were covered by the vanilla Word2vec and GloVe models, which left around 5\% of all the phenotypes with no representation. The token coverage raised to $91 \%$ when the two models were trained on PubMed, resulting in the generation of representations for $99.7 \%$ of all phenotypes. In this setting, the respective relative mean rank improvements of $11.4 \%$ and $29.7 \%$ of our approach with respect to Word2vec and GloVe should be attributed to the additional semantic information that our model introduces to the vectors as well as the more accurate representation of concepts, thanks to the disambiguation phase and the semantically constraining keywords.

For the hypernym identification task we observed a very similar trend where our model improved Word2vec and GloVe from the respective mean ranks of 1034.1 and 1021.5 to 606.1 and 556.7 on the open-domain corpus and from 317.2 and 424.9 to 277.6 and 309.5 on PubMed.

\section{Conclusions and future work}

We proposed an approach for enhancing the representation capability of existing word modeling techniques in specific domains and showed that consistent improvement can be gained over Word2vec and GloVe even when they are trained on domain-specific corpora. We plan to enhance our technique by making it sensitive to syntax and different parts of speech, such as in the manner of Baroni and Zamparelli (2010). We also plan to carry out a deeper analysis to better understand 
the potential of our model and to identify places in which it can be improved.

\section{Acknowledgments}

The authors gratefully acknowledge the support of the MRC grant No. MR/M025160/1 for PheneBank.

\section{References}

Marco Baroni and Roberto Zamparelli. 2010. Nouns are vectors, adjectives are matrices: Representing adjective-noun constructions in semantic space. In Proceedings of EMNLP, pages 1183-1193, Cambridge, Massachusetts.

Jiang Bian, Bin Gao, and Tie-Yan Liu. 2014. Knowledge-powered deep learning for word embedding. In Proceedings of the European Conference on Machine Learning and Knowledge Discovery in Databases, pages 132-148, Nancy, France.

José Camacho-Collados, Mohammad Taher Pilehvar, and Roberto Navigli. 2015. A Unified Multilingual Semantic Representation of Concepts. In Proceedings of ACL-IJCNLP, pages 741-751, Beijing, China.

Xinxiong Chen, Zhiyuan Liu, and Maosong Sun. 2014. A unified model for word sense representation and disambiguation. In Proceedings of EMNLP, pages 1025-1035, Doha, Qatar.

Nigel Collier, Anika Oellrich, and Tudor Groza. 2013. Toward knowledge support for analysis and interpretation of complex traits. Genome Biology, 14(9):111.

Manaal Faruqui, Jesse Dodge, Sujay Kumar Jauhar, Chris Dyer, Eduard Hovy, and Noah A. Smith. 2015. Retrofitting word vectors to semantic lexicons. In Proceedings of NAACL-HLT, pages 16061615, Denver, Colorado.

Juri Ganitkevitch, Benjamin Van Durme, and Chris Callison-Burch. 2013. PPDB: The paraphrase database. In Proceedings of NAACL-HLT, pages 758-764, Atlanta, Georgia.

Eric H. Huang, Richard Socher, Christopher D. Manning, and Andrew Y. Ng. 2012. Improving word representations via global context and multiple word prototypes. In Proceedings of ACL, pages 873-882, Jeju Island, Korea.

Ignacio Iacobacci, Mohammad Taher Pilehvar, and Roberto Navigli. 2015. SensEmbed: Learning sense embeddings for word and relational similarity. In Proceedings of ACL-IJCNLP, pages 95-105, Beijing, China.

Pierre Lafon. 1980. Sur la variabilité de la fréquence des formes dans un corpus. Mots, 1:127-165.
Omer Levy and Yoav Goldberg. 2014. Dependencybased word embeddings. In Proceedings of $A C L$ (Volume 2: Short Papers), pages 302-308, Baltimore, Maryland.

Tomas Mikolov, Kai Chen, Greg Corrado, and Jeffrey Dean. 2013. Efficient estimation of word representations in vector space. CoRR, abs/1301.3781.

Andrea Moro, Alessandro Raganato, and Roberto Navigli. 2014. Entity Linking meets Word Sense Disambiguation: a Unified Approach. Transactions of the Association for Computational Linguistics (TACL), 2:231-244.

Roberto Navigli and Simone Paolo Ponzetto. 2012. BabelNet: The automatic construction, evaluation and application of a wide-coverage multilingual semantic network. Artificial Intelligence, 193:217250 .

Arvind Neelakantan, Jeevan Shankar, Alexandre Passos, and Andrew McCallum. 2014. Efficient nonparametric estimation of multiple embeddings per word in vector space. In Proceedings of EMNLP, pages 1059-1069, Doha, Qatar.

Jeffrey Pennington, Richard Socher, and Christopher Manning. 2014. Glove: Global vectors for word representation. In Proceedings of EMNLP, pages 1532-1543, Doha, Qatar.

Mohammad Taher Pilehvar and Roberto Navigli. 2015. From senses to texts: An all-in-one graph-based approach for measuring semantic similarity. Artificial Intelligence, 228:95-128.

Joseph Reisinger and Raymond J. Mooney. 2010. Multi-prototype vector-space models of word meaning. In Proceedings of NAACL-HLT, pages 109117, Los Angeles, California.

Sascha Rothe and Hinrich Schütze. 2015. AutoExtend: Extending word embeddings to embeddings for synsets and lexemes. In Proceedings of ACLIJCNLP, pages 1793-1803, Beijing, China.

Christopher J. Mungall Sebastian Bauer Helen V. Firth et al. Sebastian Khler, Sandra C Doelken. 2014. The Human Phenotype Ontology project: linking molecular biology and disease through phenotype data. Nucleic Acids Research, 42:966-974.

Mo Yu and Mark Dredze. 2014. Improving lexical embeddings with semantic knowledge. In Proceedings of ACL (Volume 2: Short Papers), pages 545-550, Baltimore, Maryland. 